\title{
Antagonistic control of cell fates by JNK and p38-MAPK signaling
}

\author{
T Wada $^{1,2}$, E Stepniak ${ }^{3}$, L Hui ${ }^{3}$, A Leibbrandt ${ }^{1}$, T Katada ${ }^{4}$, H Nishina ${ }^{2}$, EF Wagner ${ }^{3}$ and JM Penninger ${ }^{\star, 1}$
}

During the development and organogenesis of all multicellular organisms, cell fate decisions determine whether cells undergo proliferation, differentiation, or aging. Two independent stress kinase signaling pathways, p38-MAPK, and JNKs, have evolved that relay developmental and environmental cues to determine cell responses. Although multiple stimuli can activate these two stress kinase pathways, the functional interactions and molecular cross-talks between these common second signaling cascades are poorly elucidated. Here we report that JNK and p38-MAPK pathways antagonistically control cellular senescence, oncogenic transformation, and proliferation in primary mouse embryonic fibroblasts (MEFs). Similarly, genetic inactivation of the JNK pathway results in impaired proliferation of fetal hepatoblasts in vitro and defective adult liver regeneration in vivo, which is rescued by inhibition of the P38-MAPK pathway. Thus, the balance between the two stress-signaling pathways, MKK7JNK and MKK3/6-p38-MAPK, determines cell fate and links environmental and developmental stress to cell cycle arrest, senescence, oncogenic transformation, and adult tissue regeneration.

Cell Death and Differentiation (2008) 15, 89-93; doi:10.1038/sj.cdd.4402222; published online 31 August 2007

Developmental programs and environmental agents trigger distinct and evolutionarily conserved kinases that relay stress signals mediating proliferation, regeneration, transformation, survival, death, senescence, or cell cycle arrest. The mitogenactivated protein kinases (MAPKs) are a family of kinases that transduce signals from the cell membrane to the nucleus in response to a wide range of stimuli, including stress. ${ }^{1-4}$ Among the MAPK signaling pathways, JNKs and p38-MAPKs were identified to be activated in response to a variety of cellular and environmental stresses such as changes in osmolarity, DNA damage, heat shock, ischemia, cytokines, UV irradiation, or oxidative stress. ${ }^{2-4} \mathrm{JNKs}$ are directly activated by the two upstream kinases MKK4 and MKK7. Once activated, JNKs regulate gene transcription via phosphorylation of transcription factors, such as Jun. ${ }^{2-5}$ In contrast, p38-MAPKs are activated by MKK3 and MKK6. ${ }^{2,4}$ These two stress pathways participate in many different intracellular signaling cascades that control a spectrum of cellular processes, including proliferation, differentiation, or apoptosis.

Although JNK and p38-MAPK have the same phylogenic origin, ${ }^{1}$ it is not known why two genetically independent stress-signaling pathways have evolved in evolution. Here we show that the two stress pathways, MKK7-JNK and MKK3/6p38-MAPK, oppositely control cellular proliferation, regeneration, senescence, and oncogenic transformation, and that qthe balance between two pathways determines cell fates in response to a variety of stress stimuli.

\section{Results}

Antagonistic effect of JNK and p38-MAPK pathways in cell proliferation. Mouse embryonic fibroblasts (MEFs) isolated from $m k k 7$ mutant embryos display impaired JNK and Jun activation, reduced cell proliferation, premature senescence, as well as augmented susceptibility to stressinduced aging (Figure 1 and Wada et al. ${ }^{6}$ ). In contrast, activation of p38-MAPK can also result in premature senescence of MEFs, ${ }^{7}$ whereas pharmacological p38MAPK inhibition induces increased cell proliferation, ${ }^{8}$ To assess functional hierarchies and potential molecular crosstalks between the JNK and p38-MAPK stress pathways in cell fate decisions, we tested the effects of p38-MAPK modulation in $m k k 7^{-1}$ MEFs. Surprisingly, inhibition of p38MAPK $\alpha / \beta$ using the selective inhibitor SB202190 restored the proliferation of $m k k 7$-deficient MEFs to that of MKK7expressing control cells (Figure 1a). To further confirm whether the reversal effects of cell proliferation are indeed p38-MAPK dependent, we introduced dominant-negative forms of MKK3 and MKK6 (MKK3-DN and MKK6-DN) into $m k k 7^{-1}$ MEFs to genetically inactivate p38-MAPK activity. Similar to SB202190, both MKK3-DN and MKK6-DN restored cell proliferation in mkk7-deficient cells (Figure 1b). Expression of MKK3-DN and MKK6-DN in MEFs inhibited UV-induced p38-MAPK activation as measured by ATF2 phosphorylation, but had no substantial effect on UV-induced JNK activation (Supplementary Figure 1a). Thus, inhibition of

\footnotetext{
${ }^{1}$ IMBA: Institute of Molecular Biotechnology of the Austrian Academy of Sciences, Dr. Bohrgasse 3, Vienna A-1030, Austria; ${ }^{2}$ Department of Developmental and Regenerative Biology, Medical Research Institute, Tokyo Medical and Dental University, 1-5-45 Yushima, Bunkyo-ku, Tokyo 113-8510, Japan; ${ }^{3}$ IMP: Institute of Molecular Pathology, Dr. Bohrgasse 7, Vienna A-1030, Austria and ${ }^{4}$ Department of Physiological Chemistry, Graduate School of Pharmaceutical Sciences, The University of Tokyo, 7-3-1 Hongo, Bunkyo-ku, Tokyo 113-0033, Japan

*Corresponding author: JM Penninger, IMBA: Institute of Molecular Biotechnology of the Austrian Academy of Sciences, Institute of Molecular Biotechnology,

Dr. Bohrgasse 3, Vienna A-1030, Austria. Tel: + 431790 44; Fax: + 43179044 110; E-mail: josef.penninger@ @imba.oeaw.ac.at

Keywords: JNK; MKK7; p38-MAPK; senescence; transformation; liver regeneration

Abbreviations: MEF, mouse embryonic fibroblast; DN, dominant negative

Received 12.12.06; revised 17.7.07; accepted 26.7.07; Edited by C Duckett; published online 31.8.07
} 


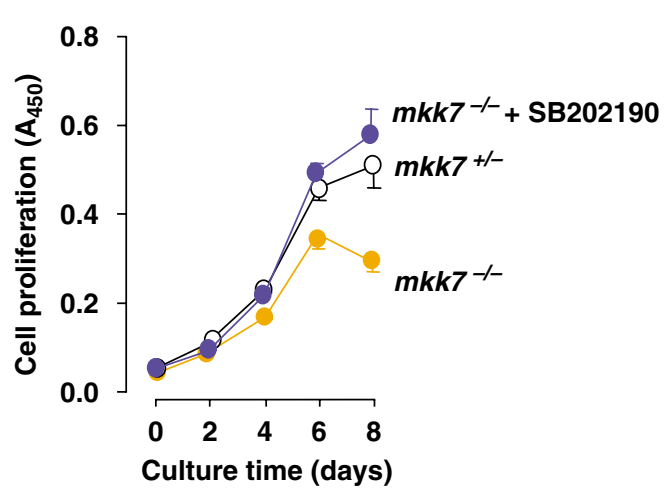

c

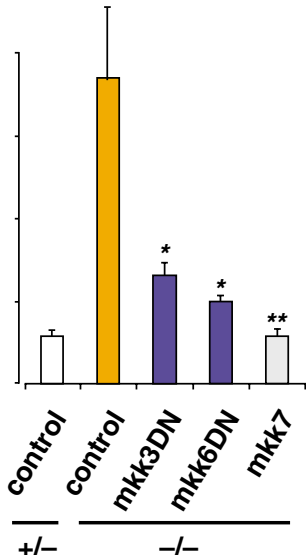

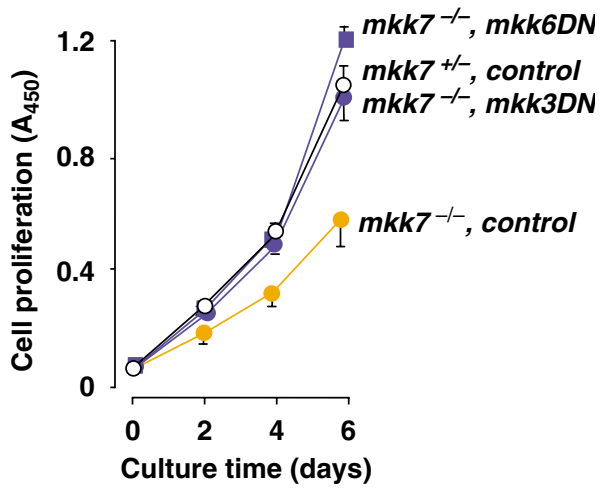

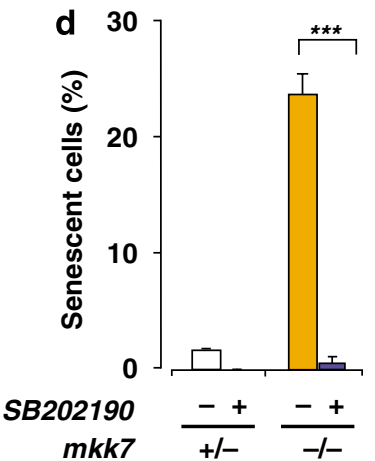

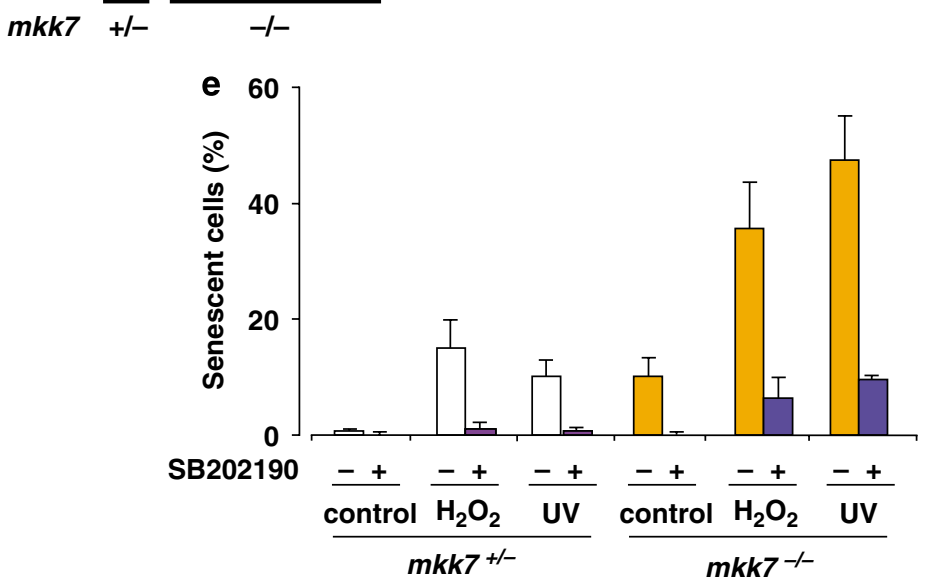

Figure 1 Antagonistic control of cell proliferation and cellular senescence by MKK7-JNK and p38-MAPK signaling. (a) Decreased proliferation of $m k k 7^{-1-}$ MEFs and rescue of the proliferation defect by the p38-MAPK inhibitor SB202190. Proliferation was determined using the cell proliferation reagent WST-1 at the indicated time points. (b) Expression of dominant-negative MKK3 (MKK3-DN) or MKK6 (MKK6-DN) restores decreased proliferation in $m k k 7^{-1}$ MEFs. An empty pBabe vector was used as control. (c, d) The MKK3/6-p38-MAPK pathway antagonizes MKK7-JNK in cellular senescence. Transfection of $m k k 7^{-1}$ MEFs with $m k k 3 D N$, mkk6DN, and wild-type $m k k 7$ as contro (c) or treatment of $m k k 7^{+/-}$and $m k k 7^{-1-}$ MEFs with the p38-MAPK $\alpha / \beta$ inhibitor SB202190 (10 $\left.\mu \mathrm{M}\right)$ (d) prevents premature senescence. Mean numbers of SA- $\beta$ galactosidase-positive senescent cells are shown. These data were confirmed using classical senescent cell morphology. ${ }^{\star} P>0.05 ;{ }^{* \star} P>0.01 ;{ }^{* \star \star} P>0.001$ (ANOVA) between control $m k k 7^{-1-}$ MEFs and $m k k 7^{-1-}$ MEFs transfected with $m k k 3 D N, m k k 6 D N$, and $m k k 7$, or after treatment with SB202190. (e) Differential roles of the JNK and p38-MAPK stress kinase pathways in environmental stress-induced senescence. $m k k 7^{+/-}$and $m k k 7^{-1}$ MEFs (passage 3) were UV irradiated $\left(10 \mathrm{~J} / \mathrm{m}^{2}\right.$ ) or treated with $\mathrm{H}_{2} \mathrm{O}_{2}$ $(150 \mu \mathrm{M})$ in the presence or absence of $10 \mu \mathrm{M} \mathrm{SB} 202190$. Stress-induced senescence (mean numbers of senescent cells determined by enlarged morphology and SA- $\beta$ galactosidase) was assayed 3 days later. Data are shown as mean \pm S.E.M.; $n=3$

the p38-MAPK pathway can rescue the proliferative defect observed in $m k k 7^{-1-}$ MEFs.

JNK and p38-MAPK antagonistically control cellular senescence. To examine whether these antagonistic effects are limited to cell proliferation, we examined other MKK7-JNK-dependent cell fate responses such as senescence. ${ }^{6}$ Treatment of $m k k 7^{-1}$ MEFs with the selective p38-MAPK $\alpha / \beta$ inhibitor SB202190, as well as overexpression of MKK3-DN and MKK6-DN indeed 
prevented premature senescence (Figure 1c and d). Treatment of wild-type cells with $\mathrm{H}_{2} \mathrm{O}_{2}$ or UV radiation resulted in premature senescence phenotype that is markedly enhanced in $m k k 7^{-1}$ MEFs (Figure 1e). Importantly, inhibition of p38MAPK $\alpha / \beta$ abolished premature senescence in both $m k k 7^{+/-}$ and $m k k 7^{-1-}$ MEFs following treatment with $\mathrm{H}_{2} \mathrm{O}_{2}$ and UV (Figure 1e). These results show that MKK7-JNK and MKK3/6p38-MAPK pathways play opposite roles in multiple cell fate responses: inactivation of the MKK7-JNK pathway results in defective proliferation and premature senescence; in contrast, inhibition of MKK3/6-p38-MAPK pathway triggers cell proliferation and markedly suppresses the cellular aging response. Importantly, inhibition of the p38-MAPK pathway allows mkk7-null cells to exit the cell cycle block and rescues $m k k 7^{-1}$ MEFs from a premature aging response in response to environmental stress.

The JNK and p38-MAPK stress pathways balance oncogenic transformation. It has been shown that cell proliferation, senescence, and cancer are closely linked at the molecular level. ${ }^{9}$ Both MKK7-JNK-Jun as well as MMK3/ MMK6-p38-MAPK signaling have been implicated in multiple cancers in humans. ${ }^{2,10}$ Since MKK7-JNK and MKK3/6-p38MAPK pathways exhibit antagonistic roles in cell proliferation and senescence, we speculated that both pathways may also exhibit opposing functions in oncogenic transformation. As reported previously, ${ }^{11}$ MEFs can be transformed by ectopic overexpression of oncogenic $\operatorname{Ras}^{12 \mathrm{~V}}$ plus c-myc (Figure $2 \mathrm{a}$ and b). Intriguingly, $m k k 7^{-1} \mathrm{MEFs}$ were markedly resistant against Ras $^{12 \mathrm{~V}}$ and c-myc-induced transformation
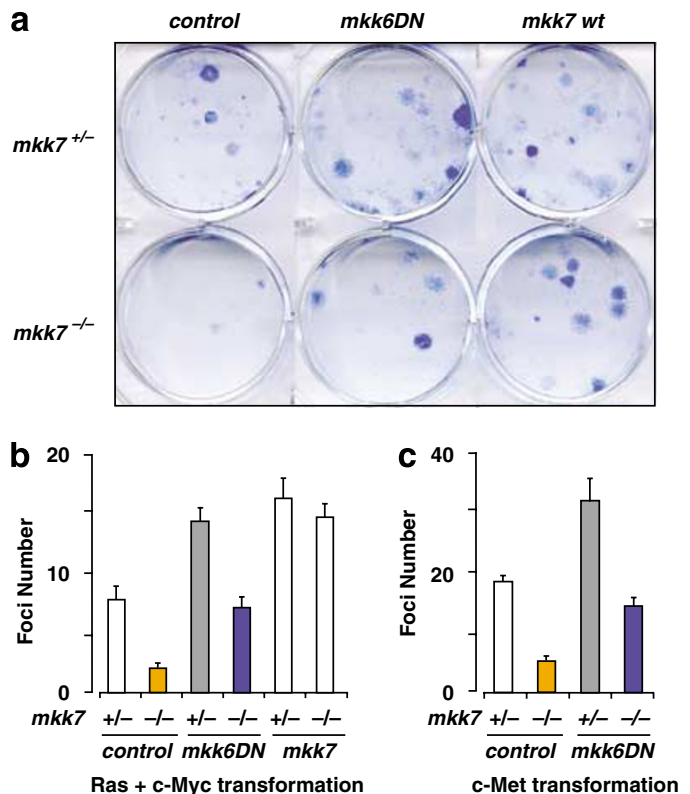

Figure 2 Opposing effect of the two stress pathways on oncogene-mediated cellular transformation. (a-c) Primary, early-passage control and $m k k 7^{-1}$ MEFs were transformed with Ras ${ }^{12 \mathrm{~V}}$ plus c-Myc or Tpr-Met in the presence or absence of MKK6-DN. Foci formation was determined on day 21. Representative appearance of foci induced by Ras ${ }^{12 \mathrm{~V}}$ and c-Myc (a) and average foci numbers in Ras ${ }^{12 \mathrm{~V}}$ - and c-Myc- (b), and Tpr-Met (c)-induced transformation are shown All data are shown as mean \pm S.E.M.; $n=3$
(Figure 2a and b). Ectopic expression of MKK7 enhanced colony formation in control MEFs and restored oncogenic transformation of $m k k 7^{-1-}$ MEFs (Figure 2a and b). p38-MAPK inhibition by MKK6-DN promoted cell transformation in control cells and, most importantly, in $m k k 7^{-1-}$ MEFs (Figure $2 a$ and b). In all our experiments p38-MAPK inhibition in $m k k 7^{1-}$ MEFs never reached transformation efficacies of MKK6-DNtransfected control MEFs or MKK7-transfected control and $m k k 7^{-1}$ MEFs. To extend these findings to another oncogenic cancer model, we examined the involvement of the MKK7-JNK and p38-MAPK pathways in Tpr-Met-induced cell transformation. ${ }^{12}$ Similar to Ras $^{12 \mathrm{~V}}$ and c-myc, overexpression of oncogenic Tpr-Met triggered transformation in control MEFs, but to a much lesser extent in mkk7-deficient cells. Importantly, suppression of the p38-MAPK via MKK6-DN again promoted cell transformation in both control MEFs and restored oncogenic transformation in mkk7-deficient MEFs (Figure 2c). These results using different oncogenes show that the MKK7JNK pathway is critical for cellular transformation, whereas p38-MAPK signaling negatively regulates oncogene-induced cellular transformation.

CDC2 is a potential target for molecular antagonisms. How can activation of two different cellular stress pathways result in opposite effects on cell cycle progression, senescence, or transformation in MEFs? We have recently identified the cell cycle kinase CDC2 as a molecular target for the MKK7-JNK signaling pathway in MEFs that links environmental stress and developmental cues to cell proliferation and senescence. ${ }^{6}$ Biochemically, CDC2 has also been identified as a downstream target for the p38-MAPK pathway. ${ }^{13}$ We therefore speculated that a molecular antagonism between these two different stresssignaling pathways might converge on CDC2. Since mkk7-deficient MEFs show reduced CDC2 expression and impaired CyclinB1 associated CDC2 kinase activity depending on the cell passage, ${ }^{6}$ we examined whether CyclinB1 associated CDC2 kinase activity can be rescued by inhibition of the p38-MAPK pathway. Whereas CDC2 expression and total CDC2 kinase activity were indeed markedly reduced in mkk7-null MEFs, inhibition of p38MAPK resulted in increased CDC2 activity, without obvious effects on CDC2 expression (Figure 3a). Inhibition of p38MAPK also resulted in increased CDC2 activity in $m k k 7^{+/-}$ MEFs, suggesting that p38-MAPK can repress CDC2 activation even in the presence of MKK7 expression (Supplementary Figure 1b). In the same experimental system, we did not observe substantially altered CyclinD1associated Cdk4 activity (Figure $3 \mathrm{~b}$ ). These results suggest that antagonistic regulation of CDC2 by MKK7-JNK and MKK3/6-p38 may be one of checkpoints by which different stress-signaling pathways control cell fate in MEFs.

Antagonism of the JNK and p38-MAPK pathways in hepatocyte proliferation and liver regeneration in vivo. To determine whether the observed molecular antagonism between MKK7-JNK and p38-MAPK pathways also occurs in other cell types, we analyzed mouse embryonic hepatoblasts. Inactivation of MKK7 in mice leads to embryonic lethality due to defective fetal liver 

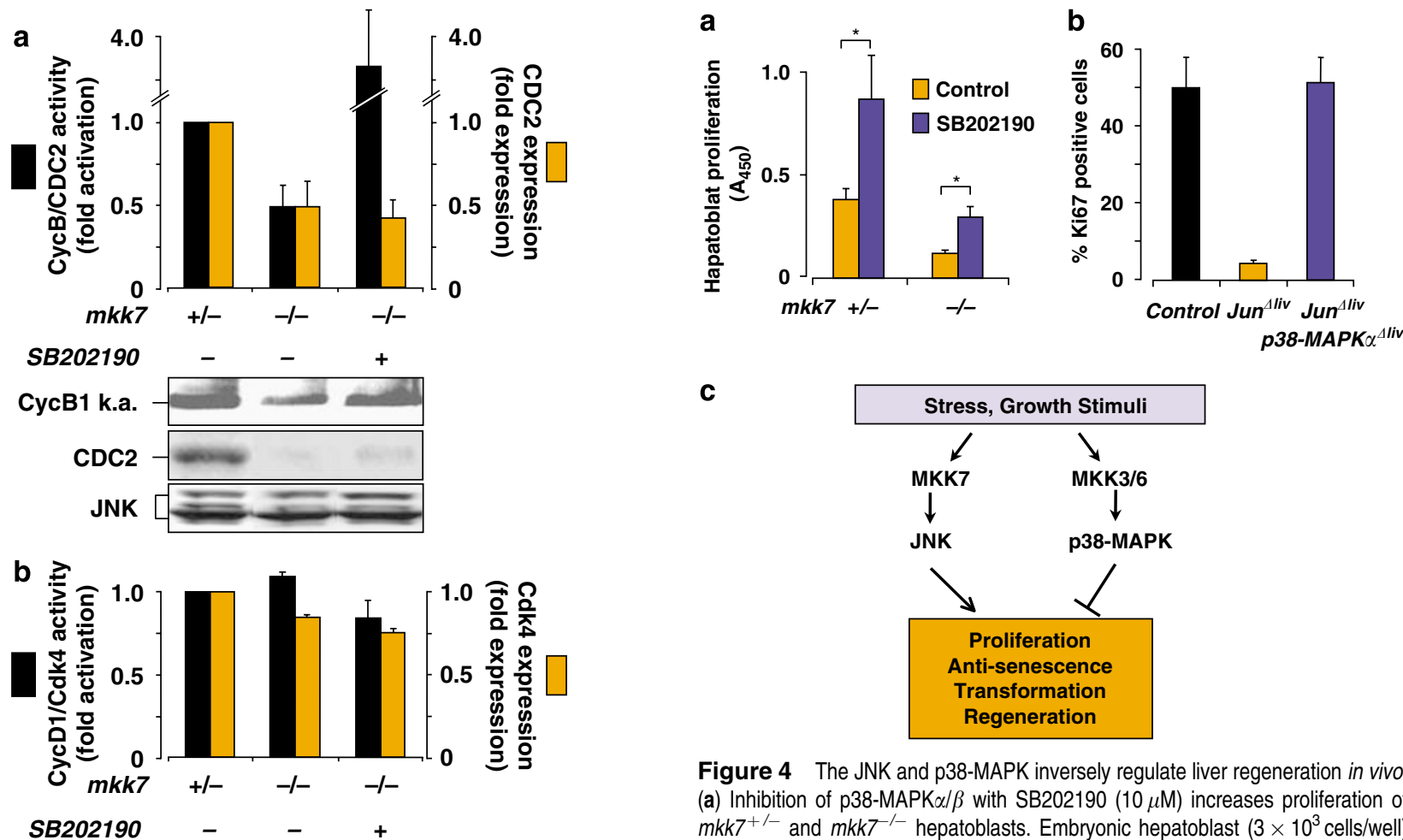

C

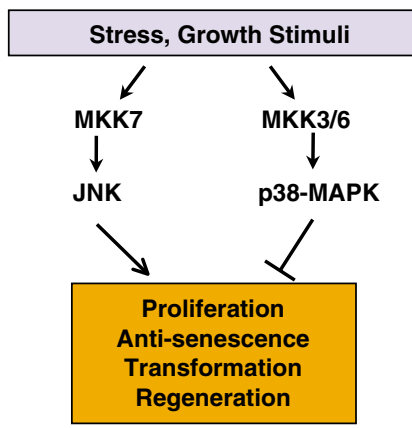

Figure 4 The JNK and p38-MAPK inversely regulate liver regeneration in vivo. (a) Inhibition of p38-MAPK $\alpha / \beta$ with SB202190 $(10 \mu \mathrm{M})$ increases proliferation of $m k k 7^{+-}$and $m k k 7^{-1}$ hepatoblasts. Embryonic hepatoblast $\left(3 \times 10^{3}\right.$ cells/well) were isolated from E11.5 $\mathrm{mkk}^{+/-}$and $\mathrm{mkk7^{-1 }}$ littermate embryos and proliferation was determined as in Figure $1 a$ and $b$. Data are shown as mean \pm S.E.M.; $n=3$. ${ }^{*} P>0.05$ (Student's $t$-test) (b) Genetic inactivation of p38MAPK $\alpha$ restores hepatocyte proliferation in jun-deficient livers after partial hepatectomy. Hepatocyte proliferation in control, Jun ${ }^{\Delta l i v}$, and Jun ${ }^{\Delta l i v} p 38-M A P K \alpha^{\Delta l i v}$ mice was determined by immunodetection of the proliferation marker Ki67 $48 \mathrm{~h}$ after partial hepatectomy. Data are shown as mean percentages of Ki67-positive hepatocytes \pm S.E.M.; $n=3$. (c) Opposing effects of the MKK7-JNK and p38MAPK stress pathways in multiple cell fate responses. Signaling via the MKK7-JNKJun pathway promotes cell proliferation, transformation, liver regeneration, and prevents cellular senescence. In contrast, the MKK3/6-p38-MAPK pathway suppresses these cell fate responses and antagonizes the JNK pathway

formation around embryonic days 11.5-13.5 (Wada et al. ${ }^{6}$ ) and impaired hepatoblast proliferation (Figure 4a). Inhibition of p38-MAPK $\alpha / \beta$ enhanced hepatoblast proliferation from control mice and rescued the impaired proliferation of $m k k 7^{-1}$ hepatoblasts (Figure 4a). To determine whether functional antagonism between the MKK7-JNK and p38-MAPK pathways is limited to embryonic cells, that is, MEFs and hepatoblasts, or if such antagonism is also operational in adult tissue, liver regeneration (as stress) after partial hepatectomy was examined. Partial hepatectomy in wildtype animals triggers liver regeneration due to rapid re-entry of hepatocytes into the cell cycle, as determined by Ki67 staining (Figure 4b). Jun is a critical downstream target of MKK7-JNK: overexpression of Jun is sufficient to rescue defective proliferation and premature senescence in $m k k 7^{-1-}$ MEFs, ${ }^{6}$ and inactivation of jun in adult mouse hepatocytes using Mx-Cre Jun ${ }^{\text {flox }}$ alleles results in defective hepatocyte proliferation (Figure $4 \mathrm{~b}$ and Behrens et al. ${ }^{14}$ ). Since p38-MAPK $\alpha$ is the predominant isoform expressed in liver (http://www.genecards.org), we therefore generated adult mice with conditional deletion of both Jun and p38-MAPK $\alpha$ $\left(M x-C r e ; J u n^{\text {floxfllox }}\right.$ p38-MAPK $\left.\alpha^{\text {flox/flox }}\right)$ in hepatocytes. Intriguingly, in vivo hepatocyte proliferation was rescued in

mice lacking both jun and $p 38-M A P K \alpha$ (Figure $4 b$ ). We next analyzed CyclinB1-associated CDC2 kinase activity. Genetic

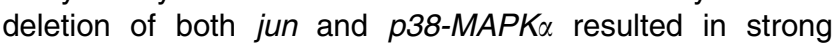
induction of CyclinB1-associated kinase activity following partial hepatectomy (Supplementary Figure 2a and b). Of note, previous experiments have also reported reduced CyclinB1-associated kinase activity in jun mutant mice. ${ }^{14}$ Consistent among all samples analyzed, markedly enhanced CyclinB1-associated CDC2 kinase activity was observed in the absence of p38-MAPK (Supplementary Figure 2, and not shown). These results show that the two stress pathways exert antagonistic effects on cell fates in embryonic and adult tissues and multiple cell types.

\section{Discussion}

Our data indicate that the two distinct stress-signaling pathways, MKK3/MKK6-p38-MAPK versus MKK7-JNK, can exert opposite effects on cell cycle progression, cellular senescence, oncogenic transformation, and adult liver regeneration (Figure 4c). In response to environmental stress and oncogenic signals, the MKK7-JNK pathway promotes 
proliferation, transformation, and regeneration and prevents premature senescence. By contrast, activation of MKK3/6p38-MAPK pathway antagonizes these MKK7-JNK functions. Although the roles of each MKK3/6-p38-MAPK and MKK7JNK pathway in cell proliferation and senescence have been previously shown by us and other groups, ${ }^{6-8}$ the molecular cross-talks and the balance of the both pathways are not well described. Our data in fibroblasts and hepatocytes suggest that the two distinct stress-signaling pathways can both act on CDC2 with opposite effects. Regulation of CDC2 by MKK7JNK and MKK3/6-p38 might be one mechanism by which stress signaling is coupled to cancer, cell cycle progression, or senescence. However, antagonistic cell fate regulations by the two stress pathways most likely also includes mechanisms other than CDC2, since many genes and proteins are regulated by MKK7-JNK-Jun and MKK3/6-p38-MAPKs. ${ }^{2}$

In conclusion, our results show that the stress kinase p38MAPK pathway antagonizes MKK7-JNK functions in terms of cellular proliferation, senescence, oncogenic transformation, and liver regeneration. These data indicate that the balance between MKK7-JNK and MKK3/6-p38-MAPK signaling determines cell fate decisions in response to environmental and developmental stress in cancer, aging, and tissue regeneration.

\section{Materials and Methods}

Mice. mkk7-deficient MEFs were obtained as described. ${ }^{6}$ To disrupt the murine Jun and p38-MAPK $\alpha$ in liver, mice carrying jun flox (Behrens et al. ${ }^{14}$ ) and p38$M A P K \alpha^{\text {flox }}$ (Engel et $a{ }^{15}{ }^{15}$ ) alleles were crossed to Mx1-Cre transgenic mice. The Cre transgene was induced in adult mice upon injections of polyinosinicpolycytidylic ribonucleic acid as described previously. ${ }^{14}$ Deletions of Jun and p38MAPK $\alpha$ were confirmed by Western blot using Jun- and p38-MAPK-specific antibodies (Cell Signaling, data not shown). Partial hepatectomy was performed as described previously. ${ }^{16}$ Mouse experiments were performed according to institutional guidelines.

MEF cultures. MEFs were prepared from E11.5. embryos and maintained in DMEM with $10 \% \mathrm{FCS}$. Since $m k k 7^{+/+}$and $m k k 7^{+/-}$MEFs behave equally in our experimental systems, $m k k 7^{+/-}$MEFs were used as controls. Cell proliferation in MEFs was determined using WST-1 (Roche). HA-tagged dominant-negative forms of MKK3 and MKK6 that carry inactivating AL mutations in their respective phosphorylation sites attenuate p38-MAPK activities, but not JNK activities in the presence of sorbitol stimuli. ${ }^{17}$ Dominant-negative MKK3 and MKK6 were ligated into a pBabe-puro vector to generate the pBabe-puro-MKK3DN and pBabe-puroMKK6DN expression constructs. The expression of MKK3DN and MKK6DN was confirmed using an anti-HA antibody (Sigma; Supplementary Figure 1a). For ectopic gene expression in MEFs, the retroviral vectors pBabe-puro as control, pBabe-puroMKK7 ${ }^{6}$, pBabe-puro-MKK3DN, or pBabe-puro-MKK6DN were transfected into phoenix-E packaging cells. MEFs were cultured in the supernatant of the packaging cells containing $5 \mu \mathrm{g} / \mathrm{ml}$ polybrene for 1 day and selected with $2.5 \mu \mathrm{g} / \mathrm{ml}$ puromycin for 2 days followed by 2 days of recovery. Empty pBabe-puro as control vector did not show any detectable effect on the phenotype of MEFs. SB202190 (Calbiochem) or the solvent DMSO were added into cultures as indicated in the figure legends. If not stated otherwise, MEFs at passages $4-5$ were used in all experiments. To detect senescent cells, SA- $\beta$-gal staining was performed (Cell Signaling). It should be noted that all SA- $\beta$-gal-positive cells had the typical flattened cell morphology characteristic of senescent cells. ${ }^{18}$

Oncogenic transformation. MEFs were transformed with oncogenic RasV12 plus c-Myc or with oncogenic Tpr-Met using the retroviral vector pBabe as described above. Three thousand cells at passage 4 were plated on six-well plates and cultured for 21 days. The resultant foci were stained with Coomassie brilliant blue, and the numbers of foci were determined.
Kinase assays. To detect Cyclin-associated kinase activities, liver samples or MEFs (passages 2-4) were homogenized in lysis buffer containing $50 \mathrm{mM}$ Tris- $\mathrm{HCl}$ (7.5), $150 \mathrm{mM} \mathrm{NaCl}, 1 \mathrm{mM}$ EDTA, $1 \mathrm{mM}$ DTT, $0.1 \%$ Tween-20, $10 \%$ glycerol, $1 \mathrm{mM}$ $\mathrm{NaF}, 0.2 \mathrm{mM} \mathrm{Na}_{3} \mathrm{VO}_{4}$, and protease inhibitor cocktail (Roche), and CyclinD1 and CyclinB1 were immunoprecipitated from the supernatants at $4^{\circ} \mathrm{C}$ using specific antibodies (Santa Cruz). Kinase activities were determined using GST-Rb for CyclinD1 (Santa Cruz) and HistoneH1 for CyclinB1 (Roche) as substrates in the presence of $\left[\gamma^{32}\right.$ P]ATP. To detect JNK and p38-MAPK kinase activities, JNK and p38-MAPK were immunoprecipitated from MEF lysates using specific antibodies (Santa Cruz and Cell Signaling, respectively). Kinase activities were determined using GST-Jun and GST-ATF2 as substrates, in the presence of $\left[\gamma^{32}{ }^{32}\right.$ P]ATP. Protein expression levels were determined using antibodies specific to CyclinB1, CyclinD1, Cdk4 (Santa Cruz), JNK, CDC2 (Cell Signaling), and actin (Sigma).

Acknowledgements. We thank Jim Woodgett for critical comments and all members of our laboratories for fruitful discussions and suggestions. JMP is supported by grants from the Austrian National Bank, the Austrian Ministry of Science and Education, IMBA, and a EU Excellence Grant. LH is a recipient of an EMBO/Marie Curie fellowship. The IMP is supported by Boehringer Ingelheim.

\section{Competing financial interests}

The authors declare that there is no competing financial interest.

1. Caffrey DR, O'Neill LA, Shields DC. The evolution of the MAP kinase pathways: coduplication of interacting proteins leads to new signaling cascades. J Mol Evol 1999; 49: 567-582.

2. Wada T, Penninger JM. Mitogen-activated protein kinases in apoptosis regulation. Oncogene 2004; 23: 2838-2849.

3. Tibbles LA, Woodgett JR. The stress-activated protein kinase pathways. Cell Mol Life Sci 1999; 55: 1230-1254.

4. Chang L, Karin M. Mammalian MAP kinase signalling cascades. Nature 2001; 410: 37-40.

5. Eferl R, Wagner EF. AP-1: a double-edged sword in tumorigenesis. Nat Rev Cancer 2003; 3: 859-868.

6. Wada T, Joza N, Cheng HY, Sasaki T, Kozieradzki I, Bachmaier K et al. MKK7 couples stress signalling to G2/M cell-cycle progression and cellular senescence. Nat Cell Biol 2004; 6: 215-226.

7. Wang W, Chen JX, Liao R, Deng Q, Zhou JJ, Huang $S$ et al. Sequential activation of the MEK-extracellular signal-regulated kinase and MKK3/6-p38 mitogen-activated protein kinase pathways mediates oncogenic ras-induced premature senescence. Mol Cell Biol 2002; 22: 3389-3403.

8. Matsumoto T, Turesson I, Book M, Gerwins P, Claesson-Welsh L. p38 MAP kinase negatively regulates endothelial cell survival, proliferation, and differentiation in FGF-2stimulated angiogenesis. J Cell Biol 2002; 156: 149-160.

9. Dimri GP. What has senescence got to do with cancer? Cancer Cell 2005; 7: 505-512.

10. Brancho D, Tanaka N, Jaeschke A, Ventura JJ, Kelkar N, Tanaka Y et al. Mechanism of p38 MAP kinase activation in vivo. Genes Dev 2003; 17: 1969-1978.

11. Land H, Parada LF, Weinberg RA. Tumorigenic conversion of primary embryo fibroblasts requires at least two cooperating oncogenes. Nature 1983; 304: 596-602.

12. Rong S, Bodescot M, Blair D, Dunn J, Nakamura T, Mizuno K et al. Tumorigenicity of the met proto-oncogene and the gene for hepatocyte growth factor. Mol Cell Biol 1992; 12: 5152-5158.

13. Manke IA, Nguyen A, Lim D, Stewart MQ, Elia AE, Yaffe MB. MAPKAP kinase-2 is a cell cycle checkpoint kinase that regulates the $G 2 / \mathrm{M}$ transition and $S$ phase progression in response to UV irradiation. Mol Cell 2005; 17: 37-48.

14. Behrens A, Sibilia M, David JP, Mohle-Steinlein U, Tronche F, Schutz G et al. Impaired postnatal hepatocyte proliferation and liver regeneration in mice lacking $\mathrm{c}$-jun in the liver. EMBO J 2002; 21: 1782-1790.

15. Engel FB, Schebesta M, Duong MT, Lu G, Ren S, Madwed JB et al. p38 MAP kinase inhibition enables proliferation of adult mammalian cardiomyocytes. Genes Dev 2005; 19 : 1175-1187.

16. Servillo G, Della Fazia MA, Sassone-Corsi P. Transcription factor CREM coordinates the timing of hepatocyte proliferation in the regenerating liver. Genes Dev 1998; 12: 36393643

17. Zanke BW, Rubie EA, Winnett E, Chan J, Randall S, Parsons M et al. Mammalian mitogenactivated protein kinase pathways are regulated through formation of specific kinaseactivator complexes. J Biol Chem 1996; 271: 29876-29881.

18. Dimri GP, Lee X, Basile G, Acosta M, Scott G, Roskelley C et al. A biomarker that identifies senescent human cells in culture and in aging skin in vivo. Proc Natl Acad Sci USA 1995; 92: 9363-9367. 